\title{
Pleistocene Radiolaria from Leg 119 Site 738B (Sections 1H1-1H2): Systematics and Biostratigraphy
}

\author{
Girish Kumar Sharma, Sheetal Kanojia \\ Department of Geology, Kumaun University, Nainital, India \\ Email: gksharma61@yahoo.com
}

Received July 26, 2013; revised August 25, 2013; accepted September 3, 2013

Copyright (C) 2013 Girish Kumar Sharma, Sheetal Kanojia. This is an open access article distributed under the Creative Commons Attribution License, which permits unrestricted use, distribution, and reproduction in any medium, provided the original work is properly cited.

\begin{abstract}
This paper presents a record of radiolarian species from the sections (1H1-1H2) Leg 119 Site 738B of Southern Ocean region. Well diversified taxa of typical Antarctic assemblage are present in the sections. Thirty species were identified and illustrated from twenty-nine samples. On the basis of appearance, disappearance and abundance of taxa, viz. Omega and Psi zones are established and systematics of stratigraphically important species is described. These zones are equivalent to NR1 and NR2 of Upper Quaternary.
\end{abstract}

Keywords: Pleistocene Radiolaria; Systematics; Biostratigraphy; Southern Indian Ocean

\section{Introduction}

The study area lies in the southern part of Kerguelen Plateau at latitude $62^{\circ} 42.54^{\prime} \mathrm{S}$ and longitude $82^{\circ} 47.25^{\prime} \mathrm{E}$ (Figure 1). The identified species shows well preserved Antarctic assemblage collected at a water depth of 2252.5 mbsf. Twenty-nine samples from two sections (1H1-1H2) were used for this study. The total length of studied samples is $2.850 \mathrm{~m}$ (Figure 2). The Leg 119 site 738B consists of nanno fossil ooze, calcareous chalk and limestone. [1] began the study on radiolarian bearing sediments in the Southern Ocean and described twenty species from the Antarctic sector of the Indian Ocean. [2] reported some species of radiolaria from Indian Ocean sector of Antarctica. [3] studied the radiolaria from the Wilhelm II Coast of Antarctica and Kerguelen Island. [4] worked on the B.A.N.Z. Antarctic Research Expedition and collected sediment samples to carry out detail study on radiolaria. [5] described Antarctic radiolarian species from the Super family Liosphaericae. [6-17] established Neogene radiolarian biostratigraphy along with illustrations and descriptions of important taxa. [18-22] carried out the detailed study on Antarctic Neogene radiolarian biostratigraphy. [23] proposed radiolarian biostratigraphy of late Tertiary Antarctic Seas of the Southern Ocean Region. [24] marked radiolarian zones of Quaternary and Upper Tertiary deposits of middle Asia. [25-33] worked on biostratigraphy and paleoclimate of the Antarctic re- gion. [34] established Pliocene-Pleistocene radiolarian biostratigraphy and paleoclimatic history from the samples collected near the Antarctic and subtropical convergence of the Southern Ocean region. [35] studied the bottom sediments of the Bellingshausen Basin in the Antarctic Sea. [36-38] established Neogene biostratigraphy of Falkland Plateau and Weddell Sea. [39] did the study on radiolarian biostratigraphy and magnetostratigraphy of siliceous microfossils from Antarctic sediments. [40] identified two new genera and seventeen new species from the Neogene sediments of the Kerguelen plateau. [41] carried out work on the biochronology and magnetostratigraphy of the Antarctic sediments. [42] established radiolarian from Eocene to Recent biostratigraphy, biogeography, diversity, and history of Southern Ocean. [43] carried out detail study on Neogene radiolaria from ODP Legs 119 and 120. [44] worked on sections of ODP Site 745 in the Kerguelen Plateau and placed the last occurrence datum of Stylatractus universus. [45] reported Antarctic Neogene radiolaria from ODP Leg 119 and described environmental control of radiolarian diversity, evolutionary rates and taxa longevities. $[46,47]$ identified and described 83 radiolarian taxa and established two Pleistocene zones of Tasman region. [48] worked on radiolarian assemblage proxies for productivity in the 0 - $6 \mathrm{Ma}$ on DSDP Site 532 and ODP Site 1084. [49] carried out the work on radiolarian paleo-productiv- 


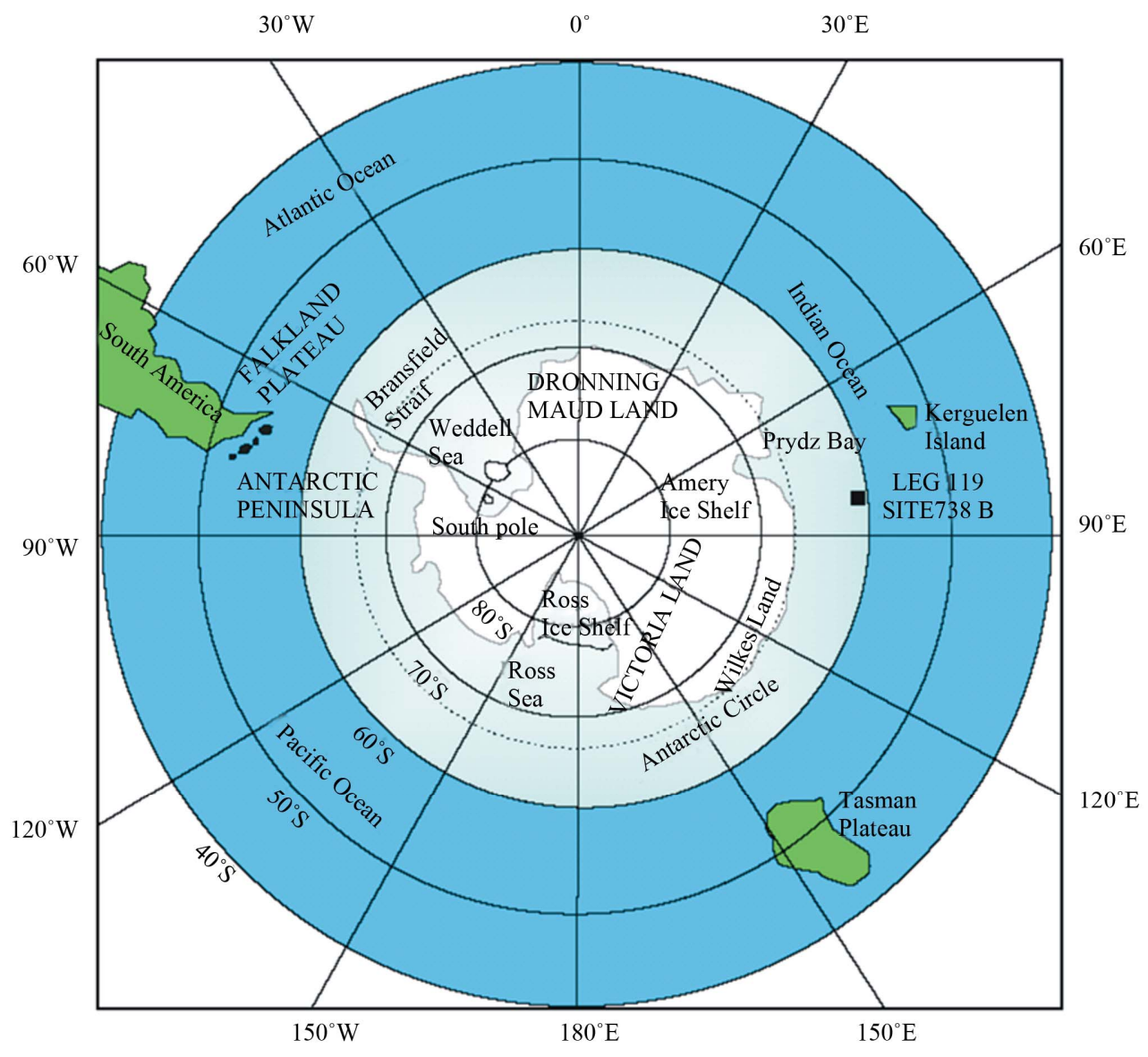

Figure 1. Showing the position of study area of Leg 119 Site 738 B.

ity variation from the ODP Site 1084 in late Pleistocene. [50,51] described 71 radiolarian species and established one radiolarian zone i.e. lower and upper Chi from the Pleistocene sediments of South East Indian area of the Antarctic continental margin. [52,53] described 45 radiolarian species and proposed the placement of NR1 and NR2 zonal boundary at a shallow depth of $6.63 \mathrm{mbsf}$ from Southern Ocean region.

\section{Methodology}

Twenty nine samples from two sections $(1 \mathrm{H} 1-1 \mathrm{H} 2)$ of Leg 119 Site $738 \mathrm{~B}$ were used to carry out the present study. Sediment samples of about 3 - $4 \mathrm{~g}$ were disaggregated in dilute Hydrogen peroxide $\left(\mathrm{H}_{2} \mathrm{O}_{2}\right)$ for $1-2$ hour followed by heating to just below the boiling point. One teaspoonful of Calgon (Hexametaphosphate) was added to further boil and disaggregate the sediment samples for 1 - 2 hours and complete the treatment. The samples were sieved through a 63 micron mesh sieve and dried. The strewn slides were prepared by using an eye dropper and Canada balsam as a mounting medium. Generally, minimum 2 - 3 slides (of cover slip size 22:22 mm) were examined for taxonomic and stratigraphic work, depend- ing on their abundance i.e. generally between 950 - 1000 individual radiolarians.

\section{Systematic Palaeontology}

The classification of the subclass Radiolaria followed here is that of [54-57]. Remarks on observed morphological features and their modifications have been added for many taxa. Species within a genus and genera within a family are arranged alphabetically. Characteristic morphological features for each new species and those given in open nomenclature are described. The synonymy for each taxon is incomplete and consists of references of interest to the present study. The distribution of each species is estimated as percentage of the total number of radiolaria present in the slides of each sample. The following scheme was followed to note the distribution-VA $=$ Very Abundant $(>50 \%) ; \mathrm{A}=$ Abundant $(20 \%-50 \%)$; $\mathrm{C}=$ Common $(5 \%-20 \%), \mathrm{F}=$ Few $(0.5 \%-5 \%), \mathrm{R}=$ Rare $(<0.5 \%)$, but more than single specimen; $+=$ Very Rare (single specimen), $-=$ absent) and preservation $\mathrm{G}=$ Good, $\mathrm{M}=$ Moderate and $\mathrm{P}=$ Poor are indicated for each sample in Table 1. The microphotographs of all the identified species are illustrated in Plate $\mathbf{I}$. 


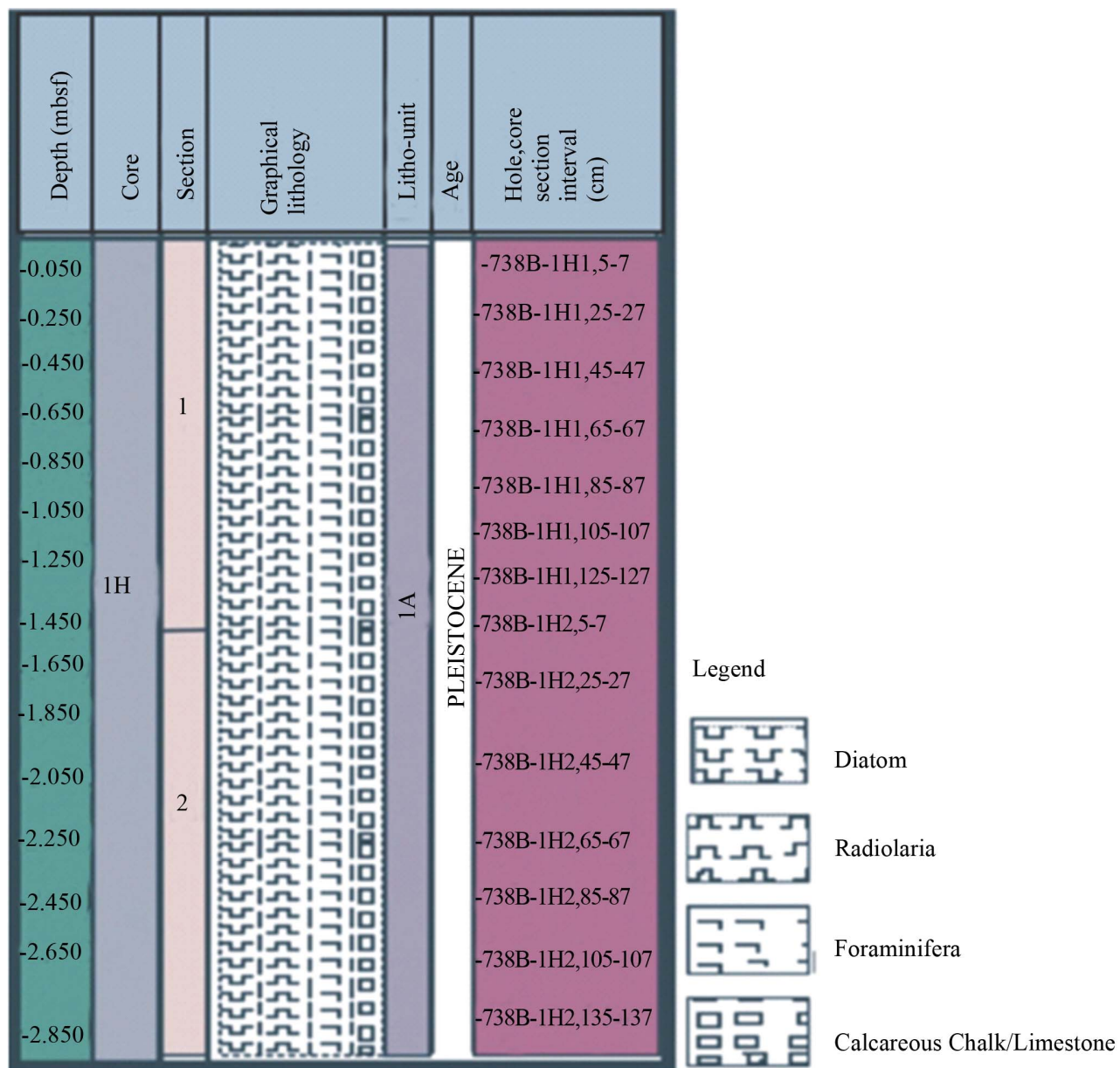

Figure 2. Showing age, position of the samples, lithostratigraphic succession and depth of Leg 119 Sites 738B.

Phylum Sarcodina Hertwig and Lesser, 1874

Class Actinopoda Calkins, 1909

Subclass Radiolaria Müller, 1858

Order Polycystina Ehrenberg, 1875, emend. Riedel, $1967 b$

Sub order Spumellaria Ehrenberg, 1875.

Family Collosphaeridae Müller, 1858

Genus Acrosphaera Haeckel, 1881

Acrosphaera sp. A

(Pl. I, Figure 1)

Abundance: Rare to Few

Distribution: [50] also showed its presence from very rare to few in the Antarctic region.

Remarks: Thick spherical lattice shell having the pores of different size and is irregularly spaced. The outer wall is slightly banded. The lattice spines vary in size from tubular to conical.

Family Actinommidae Haeckel, 1862, emend. Riedel, 1967 a

Genus Stylatractus Haeckel, 1887

Stylatractus sp.

(Pl. I, Figure 12)
Description: Ellipsoidal shell, having three concentric lattice shells. Two unequal, heavy polar spines and cylindro-conical in shape. Innermost shell spherical, thin walled with subcircular pores. Second lattice shell thick walled with subcircular pores. The shells are interconnected by radial beams and having spiny surface with large irregular pores.

Abundance: Rare

Stylatractus spp.

(Pl. I, Figure 30)

?Stylatractus neptunus Haeckel, 1887, p. 328, pl. 17, Figure 6; Riedel, 1958, p. 226, pl.1, Figure 9.

Stylatractus spp. Nigrini and Moore, 1979, p. S55, pl.

7, Figures 1(a), (b).

Abundance: Rare

Distribution: [4] considered this species as a cosmopolitan, [58] showed its distribution in the Southern Ocean region.

Stylatractus universus Hays

(P1. I, Figure 14)

Stylatractus sp. Hays, 1965, p. 167, pl. 1, Figure 6.

Stylatractus universus Hays, 1970, p. 215, pl. 1, Figures 1-2. 


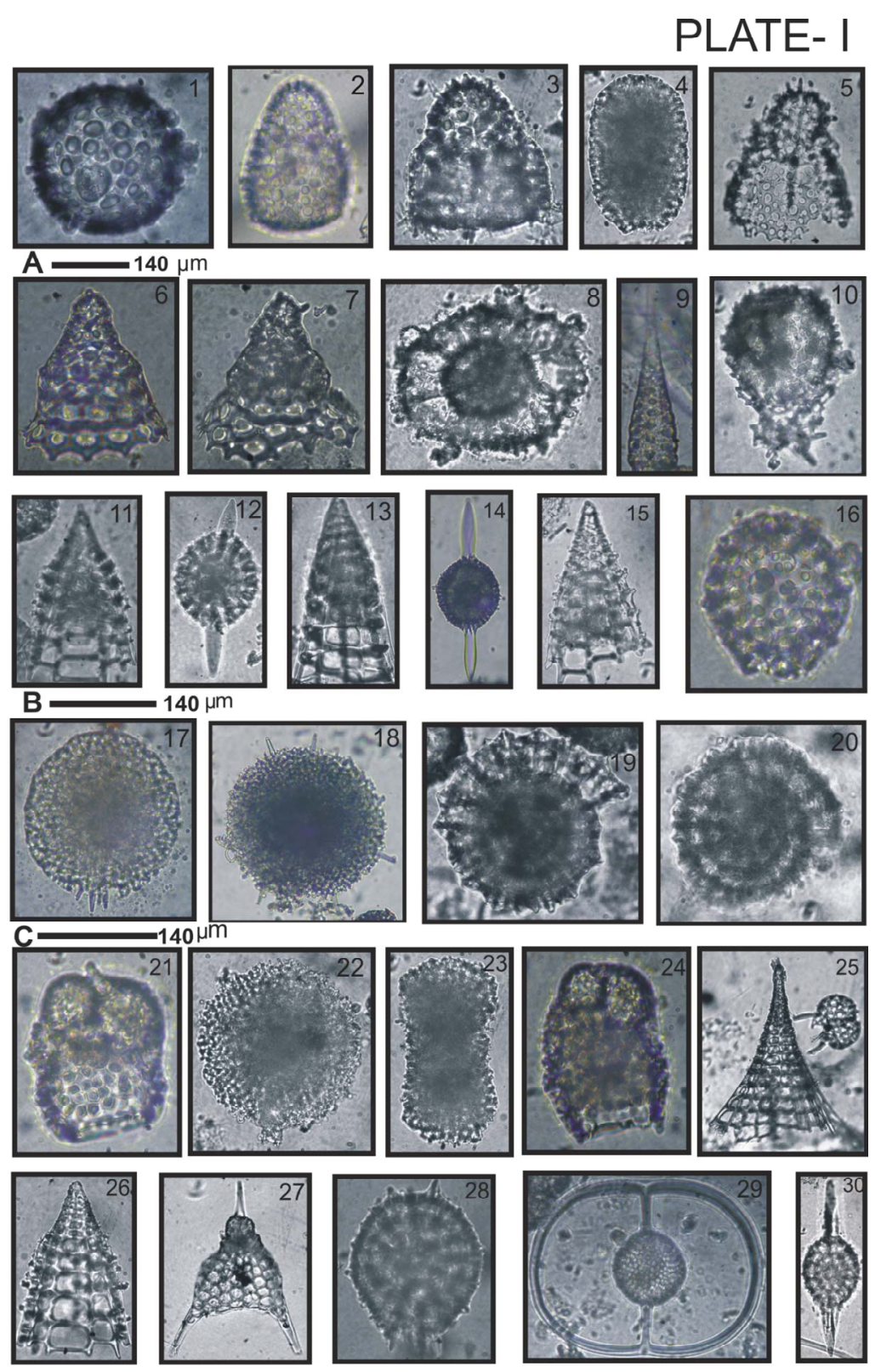

Plate I. 1. Acrosphaera sp. A, 738B-1H-1, 5-7; focused on surface. 2. Antarctissa cylindrica Ehrenberg, 738B-1H-1, 15-17; focused on outer shell. 3. Antarctissa denticulata Ehrenberg, 738B-1H-1, 15-17; focused on surface. 4. Spongurus pylomaticus Riedel, 738B-1H-1, 55-57; focused on cortical shell. 5. Antarctissa strelkovi Petrushevskaya, 738B-1H-1,115-117; focused on outer portion. 6. Cycladophora robusta Lombari and Lazarus, 738B-1H-2, 57-57; focused on surface. 7. Cycladophora davisiana Ehrenberg, 738B-1H-1, 15-17; focused on surface. 8. Phorticium pylonium (clevei) Haeckel, 738B-1H-1, 5-7; focused on cortical shell. 9. Cornutella stiligera Ehrenberg, 738B-1H-2, 15-17; focused on outer surface. 10. Mitrocalpis araneafera Reidel 738B-1H-1, 25-27; focused on outer shell. 11. Peripyramis circumtexta Haeckel, 738B-1H-1, 105-107; focused on surface. 12. Stylatractus sp., 738B-1H-1, 35-37; focused on outer portion. 13. Cinclopyramis sp. 738B-1H-1, 105-107; focused on outer part. 14. Stylatractus universus Hays, 738B-1H-1, 135-137; focused on shells. 15. Cornutella profunda Ehrenberg 738B-1H-1, 5-7; focused on surface. 16. Prunopyle sp. 738B-1H-1, 5-7; focused on outer part. 17. Spongopyle osculosa Dreyer, 738B-1H-1, 15-17; focused on surface. 18. Spongotrochus glacialis Popofsky, 738B-1H-1, 5-7; focused on surface. 19. Lithelius nautiloides Popofsky, 738B-1H-1, 15-17; focused on outer surface. 20. Lithelius minor Jörgensen 738B-1H-1, 5-7; focused on shell surface. 21. Saccospyris preantarctica Petrushevskaya, 738B-1H-1,115-117; focused on surface. 22. Spongopyle sp. 738B-1H-1, 15-17; focused on shell. 23. Spongurus sp. 738B-1H-1, 85-87; focused on outer shell. 24. Saccospyris antarctica Hackers, 738B-1H-1, 115-117; focused on outer part. 25. Plectopyramis dodecomma Haeckel, 738B-1H-1, 105-107; focused on outer periphery. 26. Bathropyramis sp. 738B-1H-1, 15-17; focused on shell surface. 27. Pterocanium charybdeum trilobum Haeckel, 738B-1H-2, 135-137; focused on surface. 28. ?Prunopyle antarctica Dreyer, 738B-1H-1, 15-17; focused on shells. 29. Saturnalis circularis Haeckel, 738B-1H-1, 105-107; focused on surface. 30. Stylatractus sp. 738B-1H-1, 5-7; focused on outer part. 
Table 1. Occurrences of radiolarian species in the samples ((a) to (d)).

(a)

\begin{tabular}{|c|c|c|c|c|c|c|c|c|c|c|c|c|c|c|c|c|}
\hline & Serial no. & 1 & 2 & 3 & 4 & 5 & 6 & 7 & 8 & 9 & 10 & 11 & 12 & 13 & 14 & 15 \\
\hline a & $\begin{array}{c}\text { Sample Name-738B } \\
\text { (Hole, Core, Section, Interval) }\end{array}$ & $\begin{array}{c}1 \mathrm{H} 1 \\
5-7\end{array}$ & $\begin{array}{l}1 \mathrm{H} 1, \\
15-17\end{array}$ & $\begin{array}{l}1 \mathrm{H} 1 \\
25-27\end{array}$ & $\begin{array}{l}1 \mathrm{H} 1 \\
35-37\end{array}$ & $\begin{array}{l}1 \mathrm{H} 1 \\
45-47\end{array}$ & $\begin{array}{l}1 \mathrm{H} 1 \\
55-57\end{array}$ & $\begin{array}{l}1 \mathrm{H} 1, \\
65-67\end{array}$ & $\begin{array}{l}1 \mathrm{H} 1 \\
75-77\end{array}$ & $\begin{array}{l}1 \mathrm{H} 1 \\
85-87\end{array}$ & $\begin{array}{l}\text { 1H1, } \\
95-97\end{array}$ & $\begin{array}{c}1 \mathrm{H} 1 \\
105-107\end{array}$ & $\begin{array}{c}1 \mathrm{H} 1 \\
115-117\end{array}$ & $\begin{array}{c}1 \mathrm{H} 1 \\
125-127\end{array}$ & $\begin{array}{c}1 \mathrm{H} 1 \\
135-137\end{array}$ & $\begin{array}{c}1 \mathrm{H} 1 \\
145-147\end{array}$ \\
\hline $\mathrm{b}$ & Depth(mbsf) & 0.05 & 0.15 & 0.25 & 0.35 & 0.45 & 0.55 & 0.65 & 0.75 & 0.85 & 0.95 & 1.05 & 1.15 & 1.25 & 1.35 & 1.45 \\
\hline $\mathrm{c}$ & Total counts & 1000 & 1000 & 980 & 1000 & 1000 & 990 & 1500 & 900 & 1113 & 900 & 1200 & 800 & 1000 & 982 & 1000 \\
\hline $\mathrm{d}$ & Age & & & $\mathrm{P}$ & $\mathrm{L}$ & $\mathrm{E}$ & I & $\mathrm{S}$ & $\mathrm{T}$ & $\mathrm{O}$ & $\mathrm{C}$ & $\mathrm{E}$ & $\mathrm{N}$ & $\mathrm{E}$ & & \\
\hline e & Abundance & $\mathrm{C}$ & $\mathrm{C}$ & $\mathrm{C}$ & $\mathrm{C}$ & $\mathrm{C}$ & $\mathrm{C}$ & $\mathrm{C}$ & $\mathrm{C}$ & $\mathrm{C}$ & $\mathrm{C}$ & $\mathrm{C}$ & $\mathrm{C}$ & $\mathrm{C}$ & $\mathrm{C}$ & $\mathrm{C}$ \\
\hline \multirow[t]{2}{*}{$\mathrm{f}$} & Preservation & $\mathrm{G}$ & $\mathrm{G}$ & G & $\mathrm{G}$ & $\mathrm{G}$ & $\mathrm{G}$ & $\mathrm{G}$ & $\mathrm{G}$ & G & G & G & $\mathrm{G}$ & G & G & G \\
\hline & Name of species & & & & & & & & & & & & & & & \\
\hline 1 & Acrosphaera sp. A & $\mathrm{F}$ & $\mathrm{R}$ & $\mathrm{R}$ & - & + & - & + & + & + & - & $\mathrm{R}$ & - & - & - & $\mathrm{R}$ \\
\hline 2 & Antarctissa denticulata & $\mathrm{C}$ & $\mathrm{C}$ & $\mathrm{C}$ & A & A & A & $\mathrm{C}$ & $\mathrm{C}$ & A & $\mathrm{A}$ & A & A & A & A & $\mathrm{C}$ \\
\hline 3 & Antarctissa cylindrica & $\mathrm{F}$ & $\mathrm{C}$ & $\mathrm{F}$ & $\mathrm{C}$ & $\mathrm{F}$ & $\mathrm{C}$ & $\mathrm{C}$ & $\mathrm{F}$ & $\mathrm{C}$ & $\mathrm{C}$ & $\mathrm{C}$ & $\mathrm{C}$ & $\mathrm{F}$ & $\mathrm{F}$ & $\mathrm{F}$ \\
\hline 4 & Antarctissa strelkovi & $\mathrm{F}$ & $\mathrm{F}$ & $\mathrm{F}$ & $\mathrm{R}$ & $\mathrm{R}$ & $\mathrm{F}$ & $\mathrm{R}$ & $\mathrm{R}$ & $\mathrm{R}$ & $\mathrm{F}$ & F & $\mathrm{F}$ & $\mathrm{R}$ & $\mathrm{F}$ & $\mathrm{F}$ \\
\hline 5 & Bathropyramis sp. & - & - & - & - & - & - & - & - & - & - & + & - & + & - & - \\
\hline 6 & Cornutella profunda & + & + & - & - & - & - & - & - & - & - & $\mathrm{R}$ & $\mathrm{R}$ & - & + & + \\
\hline 7 & Cinclopyramis sp. & - & + & - & + & - & - & - & + & - & $\mathrm{R}$ & $\mathrm{R}$ & - & $\mathrm{R}$ & $\mathrm{R}$ & + \\
\hline 8 & Cornutella stiligera & - & - & - & - & - & - & - & - & - & - & - & - & - & + & - \\
\hline 9 & Cyclodophora davisiana & - & $\mathrm{R}$ & - & + & - & - & + & - & - & - & - & + & - & + & - \\
\hline 10 & Cyclodophora robusta & - & - & - & - & - & - & - & - & - & - & - & - & - & - & - \\
\hline 11 & Lithelius minor & + & $\mathrm{R}$ & + & - & - & - & $\mathrm{R}$ & $\mathrm{R}$ & + & - & $\mathrm{F}$ & $\mathrm{R}$ & $\mathrm{R}$ & $\mathrm{R}$ & + \\
\hline 12 & Lithelius nautiloides & $\mathrm{R}$ & $\mathrm{F}$ & $\mathrm{F}$ & $\mathrm{R}$ & + & + & + & $\mathrm{R}$ & $\mathrm{F}$ & $\mathrm{R}$ & $\mathrm{F}$ & + & $\mathrm{F}$ & $\mathrm{R}$ & + \\
\hline 13 & Mitrocalpis arenifera & - & + & $\mathrm{R}$ & - & - & - & + & - & - & - & + & - & - & - & - \\
\hline 14 & Peripyramis circumtexta & - & + & $\mathrm{R}$ & - & - & - & + & - & - & - & + & - & - & - & - \\
\hline 15 & Phorticium pylonium & $\mathrm{F}$ & - & - & + & - & - & - & - & - & + & - & - & + & - & - \\
\hline
\end{tabular}

(b)

\begin{tabular}{|c|c|c|c|c|c|c|c|c|c|c|c|c|c|c|c|}
\hline & Serial no. & 16 & 17 & 18 & 19 & 20 & 21 & 22 & 23 & 24 & 25 & 26 & 27 & 28 & 29 \\
\hline $\mathrm{a}$ & $\begin{array}{c}\text { Sample Name-738B } \\
\text { (Hole, Core, Section, Interval) }\end{array}$ & $\begin{array}{c}1 \mathrm{H} 2 \\
5-7\end{array}$ & $\begin{array}{r}1 \mathrm{H} 2 \\
15-17\end{array}$ & $\begin{array}{r}1 \mathrm{H} 2 \\
25-27\end{array}$ & $\begin{array}{l}1 \mathrm{H} 2 \\
35-37\end{array}$ & $\begin{array}{l}1 \mathrm{H} 2 \\
45-47\end{array}$ & $\begin{array}{l}1 \mathrm{H} 2 \\
55-57\end{array}$ & $\begin{array}{l}1 \mathrm{H} 2 \\
65-67\end{array}$ & $\begin{array}{r}1 \mathrm{H} 2 \\
75-77\end{array}$ & $\begin{array}{r}1 \mathrm{H} 2 \\
85-87\end{array}$ & $\begin{array}{r}1 \mathrm{H} 2 \\
95-97\end{array}$ & $\begin{array}{c}1 \mathrm{H} 2 \\
105-107\end{array}$ & $\begin{array}{c}1 \mathrm{H} 2 \\
115-117\end{array}$ & $\begin{array}{c}1 \mathrm{H} 2 \\
125-127\end{array}$ & $\begin{array}{c}1 \mathrm{H} 2 \\
135-137\end{array}$ \\
\hline $\mathrm{b}$ & Depth(mbsf) & 1.55 & 1.65 & 1.75 & 1.85 & 1.95 & 2.05 & 2.15 & 2.25 & 2.35 & 2.45 & 2.55 & 2.65 & 2.75 & 2.85 \\
\hline $\mathrm{c}$ & Total counts & 1022 & 906 & 1027 & 1168 & 1062 & 1176 & 960 & 1110 & 1161 & 1193 & 1049 & 697 & 529 & 516 \\
\hline $\mathrm{d}$ & Age & & & $\mathrm{P}$ & $\mathrm{L}$ & $\mathrm{E}$ & I & $\mathrm{S}$ & $\mathrm{T}$ & $\mathrm{O}$ & $\mathrm{C}$ & $\mathrm{E}$ & $\mathrm{N}$ & $\mathrm{E}$ & \\
\hline $\mathrm{E}$ & Abundance & $\mathrm{C}$ & $\mathrm{C}$ & $\mathrm{C}$ & $\mathrm{C}$ & $\mathrm{C}$ & $\mathrm{C}$ & $\mathrm{C}$ & $\mathrm{C}$ & $\mathrm{C}$ & $\mathrm{C}$ & $\mathrm{C}$ & $\mathrm{C}$ & $\mathrm{C}$ & $\mathrm{C}$ \\
\hline \multirow[t]{2}{*}{$\mathrm{F}$} & Preservation & $\mathrm{G}$ & G & $\mathrm{G}$ & G & G & G & G & $\mathrm{G}$ & G & $\mathrm{G}$ & G & $\mathrm{G}$ & $\mathrm{G}$ & $\mathrm{G}$ \\
\hline & Name of species & & & & & & & & & & & & & & \\
\hline 1 & Acrosphaera sp. A & $\mathrm{R}$ & - & $\mathrm{R}$ & - & - & + & + & - & - & - & - & - & + & + \\
\hline 2 & Antarctissa denticulata & $\mathrm{F}$ & $\mathrm{C}$ & $\mathrm{C}$ & $\mathrm{C}$ & $\mathrm{C}$ & $\mathrm{C}$ & $\mathrm{C}$ & $\mathrm{C}$ & $\mathrm{C}$ & $\mathrm{C}$ & $\mathrm{F}$ & $\mathrm{F}$ & $\mathrm{F}$ & $\mathrm{C}$ \\
\hline 3 & Antarctissa cylindrica & $\mathrm{C}$ & $\mathrm{C}$ & $\mathrm{C}$ & $\mathrm{C}$ & $\mathrm{C}$ & $\mathrm{C}$ & $\mathrm{C}$ & $\mathrm{C}$ & $\mathrm{C}$ & $\mathrm{C}$ & $\mathrm{F}$ & $\mathrm{C}$ & F & $\mathrm{C}$ \\
\hline 4 & Antarctissa strelkovi & $\mathrm{F}$ & $\mathrm{C}$ & $\mathrm{C}$ & $\mathrm{C}$ & $\mathrm{C}$ & $\mathrm{C}$ & $\mathrm{C}$ & $\mathrm{C}$ & $\mathrm{C}$ & $\mathrm{C}$ & $\mathrm{F}$ & $\mathrm{F}$ & F & $\mathrm{C}$ \\
\hline 5 & Bathropyramis sp. & - & - & - & - & - & - & - & + & - & + & $\mathrm{F}$ & - & - & + \\
\hline 6 & Cornutella profunda & + & - & - & - & - & - & - & - & + & + & - & $\mathrm{R}$ & + & $\mathrm{R}$ \\
\hline 7 & Cinclopyramis sp. & $\mathrm{R}$ & + & - & - & - & - & - & - & + & - & + & + & - & - \\
\hline 8 & Cornutella stiligera & - & - & - & - & - & - & - & - & - & - & - & - & - & - \\
\hline 9 & Cyclodophora davisiana & - & + & - & $\mathrm{R}$ & + & $\mathrm{R}$ & $\mathrm{R}$ & + & - & - & + & + & - & $\mathrm{R}$ \\
\hline 10 & Cyclodophora robusta & - & - & - & - & - & + & - & - & - & - & - & - & - & - \\
\hline 11 & Lithelius minor & $\mathrm{R}$ & + & - & $\mathrm{R}$ & + & - & $\mathrm{R}$ & - & + & - & - & + & - & $\mathrm{R}$ \\
\hline 12 & Lithelius nautiloides & - & - & + & - & - & $\mathrm{F}$ & $\mathrm{R}$ & - & + & $\mathrm{F}$ & - & $\mathrm{F}$ & $\mathrm{R}$ & $\mathrm{R}$ \\
\hline 13 & Mitrocalpis arenifera & - & - & - & - & + & $\mathrm{R}$ & - & - & - & - & - & - & - & - \\
\hline 14 & Peripyramis circumtexta & - & - & - & - & + & $\mathrm{R}$ & - & - & - & - & - & - & - & - \\
\hline 15 & Phorticium pylonium & - & - & - & - & - & + & - & - & $\mathrm{F}$ & - & - & - & - & + \\
\hline
\end{tabular}


(c)

\begin{tabular}{|c|c|c|c|c|c|c|c|c|c|c|c|c|c|c|c|c|}
\hline & Serial no. & 1 & 2 & 3 & 4 & 5 & 6 & 7 & 8 & 9 & 10 & 11 & 12 & 13 & 14 & 15 \\
\hline $\mathrm{a}$ & $\begin{array}{c}\text { Sample Name-738B } \\
\text { (Hole, Core, Section, Interval) }\end{array}$ & $\begin{array}{c}1 \mathrm{H} 1 \\
5-7\end{array}$ & $\begin{array}{l}1 \mathrm{H} 1, \\
15-17\end{array}$ & $\begin{array}{l}1 \mathrm{H} 1 \\
25-27\end{array}$ & $\begin{array}{l}1 \mathrm{H} 1 \\
35-37\end{array}$ & $\begin{array}{l}1 \mathrm{H} 1 \\
45-47\end{array}$ & $\begin{array}{l}\text { 1H1, } \\
55-57\end{array}$ & $\begin{array}{l}\text { 1H1, } \\
65-67\end{array}$ & $\begin{array}{l}1 \mathrm{H} 1 \\
75-77\end{array}$ & $\begin{array}{l}1 \mathrm{H} 1 \\
85-87\end{array}$ & $\begin{array}{l}1 \mathrm{H} 1 \\
95-97\end{array}$ & $\begin{array}{c}1 \mathrm{H} 1 \\
105-107\end{array}$ & $\begin{array}{c}1 \mathrm{H} 1 \\
115-117\end{array}$ & $\begin{array}{c}1 \mathrm{H} 1 \\
125-127\end{array}$ & $\begin{array}{c}1 \mathrm{H} 1 \\
135-137\end{array}$ & $\begin{array}{c}1 \mathrm{H} 1 \\
145-147\end{array}$ \\
\hline $\mathrm{b}$ & Depth (mbsf) & 0.05 & 0.15 & 0.25 & 0.35 & 0.45 & 0.55 & 0.65 & 0.75 & 0.85 & 0.95 & 1.05 & 1.15 & 1.25 & 1.35 & 1.45 \\
\hline $\mathrm{c}$ & Total counts & 1000 & 1000 & 980 & 1000 & 1000 & 990 & 1500 & 900 & 1113 & 900 & 1200 & 800 & 1000 & 982 & 1000 \\
\hline $\mathrm{d}$ & Age & & & $\mathrm{P}$ & $\mathrm{L}$ & $\mathrm{E}$ & I & $\mathrm{S}$ & $\mathrm{T}$ & $\mathrm{O}$ & $\mathrm{C}$ & $\mathrm{E}$ & $\mathrm{N}$ & E & & \\
\hline $\mathrm{e}$ & Abundance & $\mathrm{C}$ & $\mathrm{C}$ & $\mathrm{C}$ & $\mathrm{C}$ & $\mathrm{C}$ & $\mathrm{C}$ & $\mathrm{C}$ & $\mathrm{C}$ & $\mathrm{C}$ & $\mathrm{C}$ & $\mathrm{C}$ & $\mathrm{C}$ & $\mathrm{C}$ & $\mathrm{C}$ & $\mathrm{C}$ \\
\hline $\mathrm{f}$ & Preservation & G & G & G & G & G & G & G & G & G & G & G & G & G & G & G \\
\hline \multicolumn{17}{|c|}{ Name of species } \\
\hline 16 & Plectopyramis dodecomma & + & - & - & - & - & + & - & - & - & - & - & - & - & + & - \\
\hline 17 & ? Prunopyle antarctica & - & $\mathrm{R}$ & - & - & - & + & - & - & + & + & + & $\mathrm{R}$ & - & - & - \\
\hline 18 & Prunopyle sp. & $\mathrm{F}$ & $\mathrm{R}$ & $\mathrm{R}$ & $\mathrm{F}$ & $\mathrm{R}$ & + & + & + & $\mathrm{R}$ & $\mathrm{R}$ & $\mathrm{R}$ & $\mathrm{R}$ & $\mathrm{R}$ & + & - \\
\hline 19 & Pterocanium c. trilobum & - & - & - & - & - & - & - & - & - & - & - & - & - & - & - \\
\hline 20 & Saccospyris antarctica & - & $\mathrm{F}$ & + & $\mathrm{R}$ & $\mathrm{F}$ & $\mathrm{F}$ & $\mathrm{R}$ & $\mathrm{F}$ & $\mathrm{F}$ & $\mathrm{F}$ & $\mathrm{F}$ & $\mathrm{F}$ & $\mathrm{F}$ & $\mathrm{F}$ & $\mathrm{F}$ \\
\hline 21 & Saccospyris preantarctica & $\mathrm{R}$ & $\mathrm{R}$ & + & $\mathrm{R}$ & $\mathrm{F}$ & + & $\mathrm{R}$ & $\mathrm{R}$ & $\mathrm{F}$ & $\mathrm{R}$ & $\mathrm{F}$ & $\mathrm{F}$ & $\mathrm{R}$ & $\mathrm{R}$ & - \\
\hline 22 & Spongopyle osculosa & $\mathrm{R}$ & $\mathrm{F}$ & $\mathrm{F}$ & $\mathrm{F}$ & $\mathrm{R}$ & $\mathrm{F}$ & $\mathrm{F}$ & $\mathrm{F}$ & $\mathrm{F}$ & $\mathrm{R}$ & $\mathrm{F}$ & $\mathrm{F}$ & $\mathrm{F}$ & + & - \\
\hline 23 & Spongopyle sp. & + & $\mathrm{R}$ & - & $\mathrm{R}$ & - & $\mathrm{R}$ & $\mathrm{R}$ & $\mathrm{R}$ & - & $\mathrm{R}$ & $\mathrm{R}$ & $\mathrm{R}$ & - & - & - \\
\hline 24 & Spongotrochus glacialis & $\mathrm{F}$ & $\mathrm{R}$ & $\mathrm{F}$ & $\mathrm{F}$ & - & $\mathrm{F}$ & $\mathrm{F}$ & $\mathrm{F}$ & $\mathrm{F}$ & $\mathrm{R}$ & $\mathrm{F}$ & $\mathrm{F}$ & $\mathrm{F}$ & - & - \\
\hline 25 & Saturnalis circularis & + & - & - & - & - & - & - & - & - & - & + & - & - & - & - \\
\hline 26 & Stylatractus spp. & - & - & - & + & + & - & + & - & $\mathrm{R}$ & - & $\mathrm{R}$ & - & + & - & - \\
\hline 27 & Stylatractus sp. & $\mathrm{F}$ & $\mathrm{R}$ & + & + & - & - & + & - & - & - & + & + & - & - & - \\
\hline 28 & Stylatractus universus & - & - & + & - & - & - & - & - & - & - & $\mathrm{R}$ & + & + & $\mathrm{R}$ & + \\
\hline 29 & Spongurus pylomaticus & $\mathrm{R}$ & $\mathrm{F}$ & $\mathrm{R}$ & $\mathrm{F}$ & - & $\mathrm{R}$ & $\mathrm{R}$ & + & $\mathrm{F}$ & $\mathrm{F}$ & $\mathrm{F}$ & $\mathrm{F}$ & $\mathrm{F}$ & $\mathrm{R}$ & $\mathrm{R}$ \\
\hline 30 & Spongurus sp. & $\mathrm{F}$ & F & $\mathrm{F}$ & $\mathrm{F}$ & $\mathrm{F}$ & $\mathrm{R}$ & $\mathrm{R}$ & $\mathrm{F}$ & $\mathrm{F}$ & $\mathrm{F}$ & $\mathrm{F}$ & $\mathrm{F}$ & F & $\mathrm{F}$ & $\mathrm{F}$ \\
\hline
\end{tabular}

(d)

\begin{tabular}{|c|c|c|c|c|c|c|c|c|c|c|c|c|c|c|c|}
\hline & Serial no. & 16 & 17 & 18 & 19 & 20 & 21 & 22 & 23 & 24 & 25 & 26 & 27 & 28 & 29 \\
\hline $\mathrm{a}$ & $\begin{array}{c}\text { Sample Name-738B } \\
\text { (Hole, Core, Section, Interval) }\end{array}$ & $\begin{array}{c}1 \mathrm{H} 2 \\
5-7\end{array}$ & $\begin{array}{l}1 \mathrm{H} 2 \\
15-17\end{array}$ & $\begin{array}{l}1 \mathrm{H} 2 \\
25-27\end{array}$ & $\begin{array}{l}1 \mathrm{H} 2 \\
35-37\end{array}$ & $\begin{array}{l}1 \mathrm{H} 2 \\
45-47\end{array}$ & $\begin{array}{l}1 \mathrm{H} 2 \\
55-57\end{array}$ & $\begin{array}{l}1 \mathrm{H} 2 \\
65-67\end{array}$ & $\begin{array}{l}1 \mathrm{H} 2 \\
75-77\end{array}$ & $\begin{array}{l}1 \mathrm{H} 2 \\
85-87\end{array}$ & $\begin{array}{l}1 \mathrm{H} 2 \\
95-97\end{array}$ & $\begin{array}{c}1 \mathrm{H} 2 \\
105-107\end{array}$ & $\begin{array}{c}1 \mathrm{H} 2 \\
115-117\end{array}$ & $\begin{array}{c}1 \mathrm{H} 2 \\
125-127\end{array}$ & $\begin{array}{c}1 \mathrm{H} 2 \\
135-137\end{array}$ \\
\hline $\mathrm{b}$ & Depth (mbsf) & 1.55 & 1.65 & 1.75 & 1.85 & 1.95 & 2.05 & 2.15 & 2.25 & 2.35 & 2.45 & 2.55 & 2.65 & 2.75 & 2.85 \\
\hline $\mathrm{c}$ & Total counts & 1022 & 906 & 1027 & 1168 & 1062 & 1176 & 960 & 1110 & 1161 & 1193 & 1049 & 697 & 529 & 516 \\
\hline d & Age & & & $\mathrm{P}$ & $\mathrm{L}$ & E & I & S & $\mathrm{T}$ & $\mathrm{O}$ & $\mathrm{C}$ & E & $\mathrm{N}$ & E & \\
\hline E & Abundance & $\mathrm{C}$ & $\mathrm{C}$ & $\mathrm{C}$ & $\mathrm{C}$ & $\mathrm{C}$ & $\mathrm{C}$ & $\mathrm{C}$ & $\mathrm{C}$ & $\mathrm{C}$ & $\mathrm{C}$ & $\mathrm{C}$ & $\mathrm{C}$ & $\mathrm{C}$ & $\mathrm{C}$ \\
\hline \multirow[t]{2}{*}{$\mathrm{F}$} & Preservation & G & G & $\mathrm{G}$ & G & G & G & $\mathrm{G}$ & G & G & $\mathrm{G}$ & G & G & G & G \\
\hline & Name of species & & & & & & & & & & & & & & \\
\hline 16 & Plectopyramis dodecomma & - & + & - & - & - & - & - & - & - & - & - & - & - & - \\
\hline 17 & ? Prunopyle antarctica & - & - & + & - & - & + & - & - & - & + & - & - & - & - \\
\hline 18 & Prunopyle sp. & $\mathrm{R}$ & - & - & + & - & $\mathrm{F}$ & $\mathrm{F}$ & - & + & + & + & + & $\mathrm{R}$ & $\mathrm{F}$ \\
\hline 19 & Pterocanium c. trilobum & - & - & - & - & - & - & - & - & - & - & - & - & - & + \\
\hline 20 & Saccospyris antarctica & $\mathrm{F}$ & $\mathrm{R}$ & $\mathrm{R}$ & $\mathrm{F}$ & $\mathrm{R}$ & $\mathrm{F}$ & $\mathrm{R}$ & $\mathrm{R}$ & $\mathrm{F}$ & $\mathrm{F}$ & - & $\mathrm{R}$ & $\mathrm{R}$ & $\mathrm{R}$ \\
\hline 21 & Saccospyris preantarctica & + & + & + & $\mathrm{R}$ & - & $\mathrm{F}$ & + & $\mathrm{F}$ & $\mathrm{R}$ & $\mathrm{R}$ & - & + & - & - \\
\hline 22 & Spongopyle osculosa & $\mathrm{R}$ & $\mathrm{R}$ & $\mathrm{R}$ & + & $\mathrm{R}$ & $\mathrm{F}$ & $\mathrm{F}$ & $\mathrm{R}$ & $\mathrm{F}$ & $\mathrm{F}$ & $\mathrm{R}$ & $\mathrm{F}$ & $\mathrm{F}$ & $\mathrm{R}$ \\
\hline 23 & Spongopyle sp. & - & - & - & + & - & - & $\mathrm{R}$ & - & - & + & - & + & + & $\mathrm{R}$ \\
\hline 24 & Spongotrochus glacialis & $\mathrm{F}$ & $\mathrm{F}$ & $\mathrm{F}$ & $\mathrm{F}$ & $\mathrm{F}$ & $\mathrm{C}$ & $\mathrm{C}$ & $\mathrm{F}$ & $\mathrm{F}$ & $\mathrm{F}$ & $\mathrm{F}$ & $\mathrm{C}$ & $\mathrm{C}$ & $\mathrm{F}$ \\
\hline 25 & Saturnalis circularis & - & - & - & - & - & - & - & - & - & - & - & + & + & + \\
\hline 26 & Stylatractus spp. & - & - & - & - & $\mathrm{R}$ & + & - & - & - & $\mathrm{R}$ & $\mathrm{R}$ & $\mathrm{R}$ & $\mathrm{F}$ & - \\
\hline 27 & Stylatractus sp. & - & - & - & + & - & + & + & - & + & $\mathrm{R}$ & - & $\mathrm{R}$ & $\mathrm{R}$ & + \\
\hline 28 & Stylatractus universus & $\mathrm{R}$ & + & - & - & + & - & - & - & - & - & - & - & - & - \\
\hline 29 & Spongurus pylomaticus & $\mathrm{F}$ & $\mathrm{R}$ & $\mathrm{R}$ & $\mathrm{F}$ & $\mathrm{F}$ & + & $\mathrm{R}$ & $\mathrm{F}$ & $\mathrm{F}$ & $\mathrm{F}$ & $\mathrm{F}$ & $\mathrm{R}$ & - & - \\
\hline 30 & Spongurus sp. & $\mathrm{F}$ & $\mathrm{F}$ & $\mathrm{F}$ & $\mathrm{C}$ & $\mathrm{C}$ & $\mathrm{C}$ & $\mathrm{F}$ & $\mathrm{C}$ & $\mathrm{C}$ & $\mathrm{F}$ & $\mathrm{F}$ & $\mathrm{F}$ & $\mathrm{F}$ & $\mathrm{F}$ \\
\hline
\end{tabular}


Abundance: Rare

Range: [9] reported its consistent presence in the Pleistocene sediments, whereas [11] showed its presence in Neogene.

Family Saturnalidae Deflandre, 1953

Genus Saturnalis Haeckel, 1881

Saturnalis circularis Haeckel

(Pl. I, Figure 29)

Saturnalis circularis Haeckel, 1887, p. 131.

Abundance: Very rare

Range: [7,12] reported its occurrence from Oligocene to lower Pleistocene.

Distribution: [46] reported its rare presence in the Tasman region. [51] Showed its distribution and taxonomy of the Southern Ocean region.

Family Sponguridae Haeckel, 1862, emend. Petrushevskaya, 1975

Genus Spongurus Haeckel, 1860

Spongurus pylomaticus Riedel

(Pl. I, Figure 4)

Spongurus pylomaticus Riedel, 1958, p. 226, pl. 1, Figures 10, 11.

Abundance: Rare to Few

Range: Neogene [12].

Spongurus sp.

(Pl. I, Figure 23)

Abundance: Rare to Common

Remark: Here, this species include only the spongy forms.

Family Spongodiscidae Haeckel, 1862, emend. Riedel, 1967b

Genus Spongopyle Dreyer, 1889

Spongopyle osculosa Dreyer

(P1. I, Figure 17)

Spongopyle osculosa Dreyer, 1889, p. 42, pl. 11, Figures 99, 100.

Abundance: Rare to Few

Range: $[7,12]$ reported its presence in Neogene.

Distribution: [46] reported its presence from rare to abundant in Tasman region. [51] showed its distribution and taxonomy of the Southern Ocean region.

Spongopyle sp.

(Pl. I, Figure 22)

Description: Spongy, biconvex shell. Shape more or less circular, central part thickened and having dense spongy mass compared to marginal part. Mantle and radial spines absent. Pylome distinct and funnel shaped.

Abundance: Rare to Few

Genus Spongotrochus Haeckel, 1860

Spongotrochus glacialis Popofsky group

(Pl. I, Figure 18)

Spongotrochus glacialis Popofsky, 1908, p. 228, pl. 26,

Figure 8; pl. 27, Figure 1, pl. 28, Figure 2.

Spongotrochus glacialis Popofsky group Petrushevs- kaya, 1975, p. 575, pl. 5, Figure 8, pl. 35, Figures 1-6. Abundance: Rare to Common

Range: Miocene to Recent [14] Neogene and Oligocene? $[7,12]$

Distribution: [4] reported its occurrence in the Antarctic region while [59] reported it in the sub-Antarctic region. [60] Considered as a cosmopolitan species. [46] also reported its presence from rare to abundant in the Pleistocene sediments of Tasman region.

Family Pyloniidae Haeckel 1881

Genus Phorticium Haeckel 1881

Phorticium pylonium Haeckel

(Pl. I, Figure 8)

Phorticium pylonium Haeckel, 1887, p. 709, pl. 49, Figure 10.

Abundance: Rare

Range: Neogene.

Genus Prunopyle Dreyer, 1889

? Prunopyle antarctica Dreyer

(Pl. I, Figure 28)

? Prunopyle antarctica Dreyer, 1889, p. 24-25, pl. 5, Figure 75.

Abundance: Rare

Range: Pleistocene to Recent (Chen, 1975a)

Distribution: $[4,13]$ reported this species from Antarctic and sub polar regions. [46] reported its presence from rare to abundant from Tasman region. [58] showed its distribution and taxonomy from Southern Ocean region.

Prunopyle sp.

(Pl. I, Figure 16)

Description: Two concentric shells connected by numerous radial bars. Pores small, circular. Cortical shell thick and bears small spines, pylome large and having spines.

Abundance: Rare to Few

Family Litheliidae Haeckel, 1862

Genus Lithelius Haeckel, 1862

Lithelius minor Jörgensen

(Pl. I, Figure 20)

Lithelius minor Jörgensen, 1900, p. 65, pl. 5, Figure 24.

Abundance: Few to Rare

Distribution: [58] showed its widespread distribution near the Antarctic region. [50] also showed its presence from very rare to few in the Southern Ocean region.

Lithelius nautiloides Popofsky

(P1. I, Figure 19)

Lithelius nautiloides Popofsky, 1908, pp. 230-231, pl.

27, Figure 4.

Abundance: Few to Rare

Range: Pliocene to Recent $[7,12,23]$ reported L. nautiloides to be endemic to the present day Antarctic fauna.

Distribution: [46] showed its distribution from rare to few in the Tasman region. [51] reported its occurrence in 
the Southern Ocean region.

SUBORDER NASSELLARIA EHRENBERG, 1875

Family Plagoniidae Haeckel, 1881, emend. Riedel, $1967 \mathrm{~b}$

Genus Antarctissa Petrushevskaya, 1967

Antarctissa cylindrica Petrushevskaya

(Pl. I, Figure 2)

4-6.

Antarctissa ewingi Chen, 1974, p. 486, pl. 3, Figures

Antarctissa cylindrica Petrushevskaya, 1975, p. 591, pl. 11, Figures 19, 20.

Abundance: Few to Common

Antarctissa denticulata (Ehrenberg)

(Pl. I, Figure 3)

Lithobotrys denticulata Ehrenberg, 1844b, p. 203.

Antarctissa denticulata (Ehrenberg) Lazarus, 1990, p.

713, pl. 3, Figures 1-4.

Abundance: Few to Abundant

Range: [14] reported its range from Pleistocene to Recent whereas [7] assigned it from the Pliocene-Pleistocene. [12] reported its range from Pliocene to Recent from the Antarctic region.

Distribution: This species occurred as rare to common in Pleistocene sediments of Tasman region [46]

Antarctissa strelkovi Petrushevskaya, 1987

(Pl. I, Figure 5)

Helotholus historicosa Jörgensen Popofsky, 1908, p. 279, pl. 32, Figures 1-5; pl. 36, Figure 2.

Antarctissa strelkovi Petrushevskaya-Lazarus, 1990, p. 713, pl. 3, Figures 13-15.

Abundance: Few to Common

Range: [14] reported its occurrence from Miocene to Recent, whereas $[7,12]$ reported its presence from Pliocene to Recent.

Distribution: [46] showed its presence from rare to abundant in the Pleistocene sediments of Tasman region.

Genus Mitrocalpis Haeckel, 1881

Mitrocalpis araneafera Popofsky

(Pl. I, Figure 10)

Mitrocalpis araneafera Popofsky, 1908, p. 273, pl. 30,

Figure 11.

Abundance: Rare

Range: Pliocene to Recent [8]

Distribution: [51] reported its occurrence in the Southern Ocean region.

Family Theoperidae Haeckel, 1881, emend. Riedel, 1967b

Genus Bathropyramis Haeckel,1882

Bathropyramis sp.

(Pl. I, Figure 26)

Description: Cephalis subspherical and poreless without apical spine, Thorax conical with six to eight longitudinal bars joined by transverse bars. The pores are sub rectangular and increase in size distally.
Abundance: Very Rare

Genus Cinclopyramis Haeckel, 1879

Cinclopyramis sp.

(P1. I, Figure 13)

Description: Pyramidal shell, thorax consisting of six to eight longitudinal bars joined by the transverse bars, network double quadrangular to rectangular pores, increasing in size distally, surface smooth and covered by mantle.

Abundance: Rare

Genus Cornutella Ehrenberg, 1838, emend. Nigrini, 1967.

Cornutella profunda Ehrenberg

(Pl. I, Figure 15)

Cornutella clathrata profunda Ehrenberg, 1854, p. 241.

Cornutella profunda Ehrenberg Riedel, 1958, p. 232, pl. 3, Figures 1, 2.

Abundance: Rare

Range: [7] reported its occurrence from Oligocene to Recent while [14] reported its presence from Miocene to Recent.

Distribution: [4] considered this species as a cosmopolitan species. [46] reported its distribution from rare to common in the Tasman region.

Cornutella stiligera Ehrenberg group

(Pl. I, Figure 9)

Cornutella stiligera Ehrenberg, 1854, pl. 36, Figure 1.

Cornutella stiligera Ehrenberg group, Petrushevskaya and Kozlova, 1972a, p. 551, pl. 30, Figures 14, 15.

Abundance: Very rare

Range: Oligocene [59]

Remark: In the studied samples, it occurs as a reworked form.

Genus Cycladophora Ehrenberg, 1872b, emend. Lombari and Lazarus, 1988

Cycladophora davisiana Ehrenberg

(Pl. I, Figure 7)

Cycladophora davisiana Ehrenberg Petrushevskaya, 1967, p. 122, pl. 69, Figures 1-7.

Abundance: Rare

Distribution: [4] considered it as a cosmopolitan species and showed its greater abundance in the high latitudes than at lower latitudes. [51] reported its occurrence in the Southern Ocean.

Cycladophora robusta Lombari and Lazarus

(Pl. I, Figure 6)

Cycladophora robusta Lombari and Lazarus, 1988, p. 105, pl. 2, Figures 1-14.

Abundance: Rare

Remark: On the margin of upper and lower part of thorax a prominent shoulder and well developed abdomen present.

Genus Pterocanium Ehrenberg, 1847 
Pterocanium charybdeum trilobum Haeckel

(P1. I, Figure 27)

Pterocanium trilobum (Haeckel), Hays, 1965, pp. 177178, pl. 3, Figure 10, Nigrini (in part), 1967, pp. 71-72, pl. 7, Figure 3(a) (only).

Pterocanium charybdeum trilobum Lazarus 1985, p. 195, Figure 10.

Abundance: Very Rare

Distribution: [25] showed its presence in early Pleistocene sediments of Antarctic

Range: Pleistocene [60]

Family Acropyramididae Haeckel, 1881

Genus Peripyramis Haeckel, 1881

Peripyramis circumtexta Haeckel

(P1. I, Figure 11)

Peripyramis circumtexta Haeckel, 1887, p. 1162, pl. 54, Figure 5.

Abundance: Rare

Range: Miocene(?)-Recent [14], Neogene and Oligocene $[7,12]$

Genus Plectopyramis Haeckel, 1881

Plectopyramis dodecomma Haeckel

(P1. I, Figure 25)

Plectopyramis dodecomma Haeckel, 1887, p. 1258, p. 54, Figure 6.

Abundance: Very Rare

Range: Neogene (Keany 1979).

Family Cannobotryidae Haeckel, 1881

Genus Saccospyris Haecker, 1908

Saccospyris antarctica Haecker

(P1. I, Figure 24)

Saccospyris antarctica Haecker, 1907, p. 447, pl. 84, Figures 584, 589, 590.

Abundance: Rare to few

Range: Pliocene-Pleistocene (Petrushevskaya, 1975).

Distribution: [51] reported its occurrence in the Southern Ocean region

Saccospyris preantarctica Petrushevskaya

(P1. I, Figure 21)

Saccospyris sp. Petrushevskaya, 1972a, pl. 2, Figure 7. Saccospyris preantarctica Petrushevskaya, 1975b, p. 589, pl. 13, Figures 19, 20.

Abundance: Rare to Few

Range: Miocene to Recent [14].

\section{Radiolarian Biostratigraphy}

All the samples from the sections of Leg 119 Site 738B show good preservation, abundant and highly diverse radiolarian assemblages. The assemblage belongs to a typical Antarctic and consists of thirty taxa. The dominant species present in the sections are Antarctissa denticulata, Antarctissa cylindrica, Antarctissa strelkovi, Lithelius minor, Lithelius nautiloides, Saccospyris antarctica, Saccospyris preantarctica, Spongopyle osculosa, Spon- gotrochus glacialis, Spongurus pylomaticus and Spongurus sp. In this study, Antarctic Nogene radiolaria zonation of $[25,61]$ and $[38,45]$ are followed.Two zones has been recognised viz., Omega and Psi. [25] defined the base of the Omega zone ( $\sim 0.43$ Ma-Recent) as the last appearance of Stylatractus universus and top as Holocene. In the studied samples, Stylatractus universus is present very rare to rare from samples number $738 \mathrm{~B}$ $1 \mathrm{H} 1,25-27$ at a depth of 0.25 mbsf to $738 \mathrm{~B}-1 \mathrm{H} 2,45-$ 47 at a depth of 1.95 mbsf. However, [7] considered the zone NR1 as Antarctissa denticulata zone and defined the bottom of this zone as the last ocurrence of $S$. universus. In the studied sequence, its last appearance in the sample number 738B-1H1, $25-27$ at a depth of 0.25 mbsf and occur as very rare. The zone NR1 of [64] is considered equivalent to Omega zone of [25]. The Psi zone $(\sim 0.8-0.43 \mathrm{Ma})$ was defined by [25] as the base of last appearance of $P$. $c$ trilobum and top as the last appearance of S. universus [26].

In the studied samples, $P . c$ trilobum is present in the samples 738B-1H2, $135-137$ at a depth of $2.85 \mathrm{mbsf}$ occurs as very rare, while $S$. universus continuosly shows its presence throughout the sections. [14] considered $A$. cylindrica as a better marker within this stratigraphic interval and occur as few to common. The zone NR2 as defined by Caulet, in part, and S. universus Zone of Chen, in part as top and bottom of this zone as the last occurances of $S$. universus and Phormostichoartus pitomorphus respectively. In the studied samples, the zone NR2 is demarcated at the sample 738B-1H1, 25 27 at a depth 0.25 mbsf. Thus the total age of the sections 1H1-1H2 lies between 0.8 Ma to Recent (Figure 3).

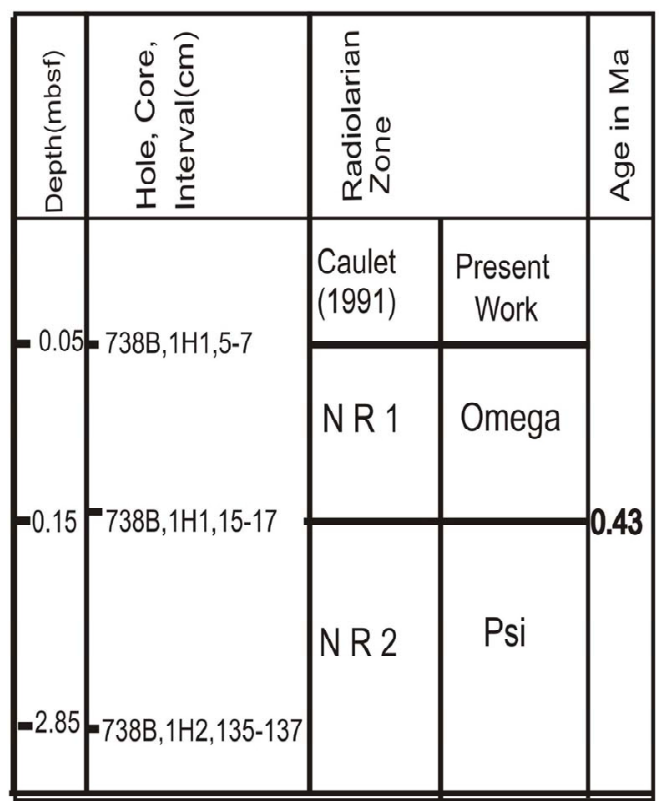

Figure 3. Showing the age and radiolarian zones of Leg 119 Site 738B, Sections 1H1-1H2. 


\section{Conclusion}

Leg 119 Site $738 \mathrm{~B}$ of sections $1 \mathrm{H} 1-1 \mathrm{H} 2$ shows the typical assemblage of Antarctic radiolaria in Upper Pleistocene $(0-0.8 \mathrm{Ma})$. Thirty radiolarian taxa were identified and their taxonomy notes of stratigraphically important taxa were given. Two radiolarian zones namely Omega and Psi were recognised on the basis of the first and the last common occurences of Upper Quaternary and are equivalent to NR1 and NR2 respectively. Further, the zonal boundary is marked at a depth of $0.15 \mathrm{mbsf}$.

\section{Acknowledgements}

This study was made possible while one of the auther (GKS) got the samples from the Lamont-Doherty Geological Observatory, USA (Request Number-18214 A). We are thankful and grateful to UGC, New Delhi, India (Project no.SR/F.No.34-42\2008) for providing the financial assistance in the form of major project to one of the author (GKS). We are also thankful to Head, Department of Geology for providing the facilities.

\section{REFERENCES}

[1] C. G. Ehrenberg, "Einige vorlaufige Resultate seiner Untersuchungen der ihm von der Sudpolreise des Capitain Ross, so wie von den Herren Schayer und Darwin ZugekoMmenen Materialien uber das Verhalten," Monatsber. Königliche Preussischen Akademie der Wissenschaften zu Berlin Jahre, Vol. 1844, 1844, pp. 182-207.

[2] E. Haeckel, "Report on the Radiolaria Collected by H.M.S. Challenger during the Years 1873-1876. Reports of Scientific Results," Voyage H.M.S. Challenger, Zoology, Vol. 18, 1887, pp. 1-1803.

[3] A. Popofsky, "Die Radiolarien des Antarktis (mit Ausnahme der Tripyleen)," Deutsche Südpolar-Expedition 1901-1903, Part 10, Zoology, Vol. 2, No. 3, 1908, pp. 183-305.

[4] W. R. Riedel, "Radiolaria in Antarctic Sediments," Reports B.A.N.Z. Antarctic Research Expedition, Vol. 6, No. 10, 1958, pp. 217-255.

[5] K. Nakaseko, "On Superfamily Liosphaericae (Radiolaria) from Sediments in the Sea near Antarctica, Part 1. On Radiolaria from Sediments in the Sea near Antarctica," Special Publication from the Seto Marine Biology Laboratory, Biological Results of the Japanese Antarctic Expedition, Vol. 2, 1959, pp. 1-21.

[6] P. H. Chen, "Some New Tertiary Radiolaria from Antarctic Deep Sea Sediments," Micro-Paleontology, Vol. 204, 1974, pp. 480-492. http://dx.doi.org/10.2307/1485135

[7] P. H. Chen, “Antarctic Radiolaria,” In: D. E. Hayes, L. A. Frakes, et al., Eds., Initial Reports of Deep Sea Drilling Project, US Government Printing Office, Washington DC, Vol. 28, 1975, pp. 437-513.

[8] P. H. Chen, "Post Palaeocene Radiolaria: Their Taxonomy, Biostratigraphy and Phylogeny and Development of Late Neogene Cold Water Faunas," Ph.D. Dissertation,
Columbia University, New York, 1975.

[9] J. D. Hays and N. D. Opdyke, "Opdyke Antarctic Radiolaria, Magnetic Reversal and Climatic Change," Science, Vol. 158, 1967, pp. 1001-1011.

[10] J. Keany and J. P. Kennett, "Pliocene-Early Pleistocene Paleoclimatic History Recorded in Antarctic-Subantarctic Deep-Sea Cores," Deep Sea Research, Vol. 19, No. 8, 1972, pp. 529-548.

[11] J. Keany, "Early Pliocene Radiolarian Biostratigraphy of the Southern Ocean," US Antarctic, Vol. 11, 1976, pp. 171-173.

[12] J. Keany, "Early Pliocene Radiolarian Taxonomy and Biostratigraphy in the Antarctic Region," Micropaleontology, Vol. 25, No. 1, 1979, pp. 50-74. http://dx.doi.org/10.2307/1485210

[13] M. G. Petrushevskaya, "Radiolyarii otryadov Spumellaria i Nassellaria Antarkticheskoi Oblasti," In: Issledovaniya Fauny Morei, t. IV (XII), Resultaty Biologicheskikh Issledo vanii Sovetskoi Antarkticheskoi, Ekspeditsii 1955-1958, Vol. 3, 1967, pp. 5-186.

[14] M. G. Petrushevskaya, "Cenozoic Radiolarians of the Antarctic, Leg 29, DSDP,” In: J. P. Kennett, R. E. Houtz, et al., Eds., Initial Reports of Deep Sea Drilling Project, US Government Printing Office, Washington DC, Vol. 29, 1975, pp. 541-675.

[15] F. M. Weaver, "Antarctic Radiolaria from the Southern Pacific Basin, DSDP, Leg35," In: C. D. Hollister, C. Craddock, et al., Eds., Initial Reports of Deep Sea Drilling Project US Government, Printing Office, Washington DC, Vol. 35, 1976, pp. 569-603.

[16] F. M. Weaver, "Late Miocene to Pliocene Radiolarian Paleobiogeography and Bio-Stratigraphy of the Southern Ocean," Ph.D. Dissertation, Florida State University, Tallahassee, 1976.

[17] G. Lombari and D. B. Lazarus, "Neogene Cycladophorid Radiolaria from North Atlantic, Antarctic and North Pacific Deep Sea Sediments," Micropaleontology, Vol. 34, No. 2, 1988, pp. 97-135. http://dx.doi.org/10.2307/1485657

[18] W. R. Riedel and A. Sanfilippo, "Radiolaria Leg 4, DSDP," In: R. G. Bader, et al., Eds., Initial Report of Deep Sea Drilling Project, US Government Printing Office, Washington DC, Vol. 4, 1970, pp. 503-573.

[19] W. R. Riedel and A. Sanfilippo, "Cenozoic Radiolaria from the Western Tropical Pacific, Leg 7, p.1529-1672," In: E. L. Winterer, et al., Eds., Initial Reports of Deep Sea Drilling Project, US Government Printing Office, Washington DC, Vol. 7, 1971, pp. 503-575.

[20] A. S. Kling, "Radiolaria: Leg 6 of Deep Sea Drilling Project," In: Initial Reports of Deep Sea Drilling Project, US Government Printing Office, Washington DC, Vol. 6, 1971, pp. 1069-1117.

[21] F. Theyer, C. Y. Mato and S. R. Hammond, "Paleomagnetic and Geochronologic Calibbration of Latest Oligocene to Pliocene Radiolarian Events," Equatorial Pacific Marine Micropaleontology, Vol. 3, 1978, pp. 377-395. http://dx.doi.org/10.1016/0377-8398(78)90019-1

[22] W. R. Riedel and M. J. Westberg, "Neogene Radiolarians 
from the Eastern Tropical Pacific and Caribbean, DSDP Leg 68, p.289-300," In: Initial Reports of Deep Sea Drilling Project, US Government Printing Office, Washington DC, Vol. 68, 1982, pp. 289-300.

[23] J. D. Hays, "Radiolaria and Late Tertiary and Quaternary history of Antarctic Seas." In: Biology of Antarctic Seas II, American Geophysical Union, Antarctic Research Series, Vol. 5, 1965, pp. 125-184.

[24] M. G. Petrushevskaya, "On the Natural System of Polycystine Radiolaria (Class Sarcodina)," In: A. Farinacci, Ed., Proceedings of the II Plantonic Conference, Edizioni Tecnosclenza, Roma, 1971, pp. 981-992.

[25] J. D. Hays, "Quaternary Sediments of Antarctic Ocean," Progress in Oceanography, Vol. 4, 1967, pp. 117-131. http://dx.doi.org/10.1016/0079-6611(65)90045-5

[26] J. D. Hays, "Stratigraphy and Evolutionary Trends of Radiolaria in North Pacific Deep-Sea Sediments," In: J. D. Hays, Ed., Geological Investigations of the North Pacific, Geological Society of America, Memoir, Vol. 126, 1970, pp. 185-218. http://dx.doi.org/10.1130/MEM126-p185

[27] O. L. Bandy and R. E. Casey, "Major Late Cenozoic Planktonic Datum Planes, Antarctic to the Tropics," Antarctica, Vol. 5, No. 4, 1969, pp. 170-171.

[28] J. D. Hays and W. A. Berggren, "Quaternary Boundaries and Correlations," B. M. Funnell and W. R. Riedel, Eds., Micropaleontology of the Oceans Cambridge University Press, Cambridge, 1971, pp. 669-691.

[29] M. G. Petrushevskaya and G. E. Kozlova, "Radiolaria: Leg 14, DSDP,” In: D. E. Hayes, A. C. Pimm, et al., Eds., Initial Reports Deep Sea Drilling Project, US Government Printing Office, Washington DC, Vol. 24, 1972, pp. 495-648.

[30] M. G. Petrushevskaya, "Some Aspects of Paleogeography Based on the Radiolarian Analysis of the Deep Sea Bottom Sediments," Okeanologia, Vol. 12, No. 4, 1972, pp. 640-653.

[31] M. G. Petrushevskaya, "Polycystine Radiolarians in the Bottom Sediments of the Indian Ocean," Okeanologia, Vol. 13, No. 6, 1973, pp. 1041-1051.

[32] M. G. Petrushevskaya, "Radiolarian Biostratigraphy of Neogene Deep-Sea Sediments of Antarctica," In: A. P. Zhuze, Ed., Marine Micropaleontology (Diatoms, Radiolarians, Silicoflagellates, Foraminifers and Calcareous Nannoplankton), Nauka, Moscow, 1978, pp. 82-90.

[33] F. M. Weaver, "Correlation of Late Miocene-Early Pliocene Radiolarian Zones to the Paleomagnetic Time Scale," Antarctic US, Vol. 10, No. 5, 1975, pp. 270-271.

[34] J. Keany and J. P. Kennett, "Pliocene-Pleistocene Radiolarian Biostratigraphy and Paleoclimatology at DSDP Site 278 on the Antarctic Convergence," In: Initial Reports of Deep Sea Drilling Project, US Government Printing Office, Washington DC, Vol. 29, 1975, pp. 757-767.

[35] K. Nakaseko and A. Nishimura, "Radiolaria from the Bottom Sediments of the Bellingshausen Basin in the Antarctic Sea," Report of the Technology Research Center, Japan National Oil Corporation, Vol. 16, 1982, pp. 91-244.
[36] F. M. Weaver, "Cenozoic Radiolarians from the SouthWest Atlantic, Falkland Plateau Region, DSDP Leg 71," In: W. J. Ludwig, V. A. Krasheninnikov, I. A. Basov, U. Bayer, J. Bloemendal, B. Bornhold, P. F. Ciesielski, E. H. Goldstein, C. Robert, J. Salloway, J. L. Usher, H. von der Dick, F. M. Weaver and S. W. Wise Jr., Eds., Initial Reports of the Deep Sea Drilling Project 71, US Government Printing Office, Washington DC, 1983, pp. 667-686. http://dx.doi.org/10.2973/dsdp.proc.71.126.1983

[37] A. Abelmann and R. Gersonde, "Cycladophora davisiana stratigraphy in Pliocene-Pleistocene Cores from the Antarctic Ocean (Atlantic Sector)," Micropaleontology, Vol. 34, No. 3, 1988, pp. 268-276. http://dx.doi.org/10.2307/1485757

[38] D. Lazarus, "Middle Miocene to Recent Radiolarians from the Weddell Sea, Antarctica, ODP Leg 113," In: P. F. Barkar and J. P. Kennett, Eds., Proceedings of Ocean Drilling Program Scientific Results, 1990, pp. 709-728.

[39] R. Gersonde, A. Abelmann, L. H. Burckle, N. Hamilton, D. Lazarus, K. MC Cartney, P. O'Brien, V. Spiess and S. W. Wise Jr., "Biostratigraphic Synthesis of Neogene Siliceous Microfossils from the Antarctic Ocean, ODP Leg 113 (Weddell Sea)," In: P. F. Barker, J. P. Kennett, S. O'Connell, S. Berkowitz, W. R. Bryant, L. H. Burckle, P. K. Egeberg, D. K. Fiitterer, R. E. Qersonde, X. Qolovchenko, N. Hamilton, L. Lawver, D. B. Lazarus, M. Lonsdale, B. Mohr, T. Nagao, C. P. Q. Pereira, C. J. Pudsey, C. M. Robert, E. Schandl, V. Spiej, L. D. Stott, E. Thomas, K. F. M. Thompson and S. W. Wise Jr., Eds., Proceedings of the Ocean Drilling Program, Scientific Results. College Station, TX (Ocean Drilling Program), 1990, pp. 915-936.

[40] J. P. Caulet, "Radiolaria from the Kerguelen Plateau, Leg 119," In: J. Barron, B. Larsen, et al., Eds., Proceedings of the Ocean Drilling Program Scientific Results, Vol. 119, pp. 513-542.

[41] J. A. Barron, J. G. Baldauf, E. Barrera, J. P. Caulet, B. T. Huber, B. H. Keating, D. Lazarus, H. Sakai, H. R. Theirstein and W. Wei, "Biochronologic and Magnetographic Synthesis of Leg119 Sediments from the Kerguelen Plateau and Prydz Bay," J. A. Barron and B. Larsen, Eds., Antarctica, 1991.

[42] D. Lazarus and J. P. Caulet, "Eocene to Recent Radiolarian Biostratigraphy, Bio Geography, Diversity, and History of the Southern Oceans," Internation Conference on the Role of the Southern Oceans. International Conference on the Role of the Southern Ocean and Antarctic global Change: An Ocean Drilling Perspective, Santa Barbara, 1991, Abstract, p. 30.

[43] D. Lazarus, "Antarctic Neogene radiolarians from the Kerguelen Plateau, ODP Legs 119 \&120,” In: S. W. Wise and R. Schlich, Eds., Proceedings of Ocean Drilling Program Scientific Results, Leg 120, 1992, pp. 785-810.

[44] L. Mcintyre and I. Kaczmarska, "Improved Resolution of the Pleistocene Extinction Level of Stylatractus universus (Hays) in ODP Hole 75B, Kerguelen Plateau," Micropaleontology, Vol. 42, No. 4, 1996, pp. 375-379. http://dx.doi.org/10.2307/1485959

[45] D. Lazarus, "Environmental Control of Diversity, Evolutionary Rates and Taxa Longevities in Antarctic Neogene 
Radiolarian," Paleontologica Electronica, Vol. 5, 2002, p. 32

[46] G. K. Sharma, K. Takahashi and V. S. Dalakoti, "Taxonomy and Distribution of Pleistocene Radiolarians from the Tasman Region," Neues Jahrbuch Für Geologie und Paläontologie, Vol. 231, 2004, pp. 297-347.

[47] G. K. Sharma, K. Takahashi and V. S. Dalakoti, "Biostratigraphy and Distribution of Pleistocene Radiolaria from the Tasman Region," Acta Micropalentologica Sinica, Vol. 23, No. 1, 2006, pp. 31-50.

[48] B. Bittnipok, D. Lazarus, L. Diester-Haass, P. Meyers, P. and K. Billups, "Radiolarian Assemblage Proxies for Productivity in the 0 - 6 Ma Benguela Upwelling System, DSDP Site 532 and ODP Site 1084," Programs and Abstracts, Third Joint Meeting of the Palynology and Silicofossil groups of the Micropalaeontological Society, Utrecht, 9-10 March 2006, p. 28.

[49] G. K. Sharma and K. Takahashi, "Pleistocene Radiolaria from the Antarctic Continental Margin: Distribution and Biostratigraphy," Geological Society of India, Vol. 69, 2007, pp. 813-826.

[50] G. K. Sharma and K. Takahashi, "Distribution and Taxonomy of Pleistocene Radiolarians from the SE Indian Area of the Antarctic Continental Margin Paleoworld," Journal of Paleontological Research, Vol. 17, 2008, pp. 57-82.

[51] G. K. Sharma and J. Bora, "Pleistocene Radiolaria from the Kerguelen Plateau, Leg 119, Sections (1H-2H)," Palaeontological Society of India, Vol. 52, No. 1, 2007, pp. 27-39.

[52] G. K. Sharma and J. Bora, "Pleistocene Radiolarian from the Leg 119 Site 745, Section $(1 \mathrm{H}-4 \mathrm{H})$ in the Southern Ocean Region," Geological Society of India, Vol. 73, No. 2, 2009, pp. 165-177.
[53] C. A. Nigrini and T. C. Moore Jr., "A Guide to Modern Radiolaria," Cashman Foundation for Foraminiferal Research Special Publication, 1979, pp. S1-142, N1-106.

[54] C. A. Nigrini and C. Lombari, "Miocene Radiolaria," Cushman Foundation for Foraminiferal Research Special Publication, 1984, pp. S1-102, N1-206.

[55] D. Lazarus, "Middle Miocene to Recent Radiolarians from the Weddell Sea, Antarctica, ODP Leg 113," In: P. F. Barkar and J. P. Kennett, Eds., Proceedings of Ocean Drilling Program Scientific Results, 2007, pp. 709-728.

[56] G. Lombari and G. Boden, "Modern Radiolarian Global Distribution," Cushman Foundation for Foraminiferal Research Special Publication, 1985, pp. 1-125.

[57] J. A. Lozano, "Antarctic Sedimentary, Faunal and Sea Surface Temperature Responses during the Last 230,000 Years with Emphasis in Comparison between 18,000 Years ago and Today," Ph.D. Dissertation, Columbia University, New York, 1974, p. 400.

[58] R. N. Benson, "Recent Radiolaria from the Gulf of California," Ph.D. Dissertation, Minnesota University, 1966. pp. 577, pls. 1-35, text-figs. 1-12, tables 1-2.

[59] M. G. Petrushevskaya and G. E. Kozlova, "Radiolaria: Leg 14, DSDP,” In: D. E. Hayes, A. C. Pimm, et al., Eds., Initial Reports Deep Sea Drilling Project, US Government Printing Office, Washington DC, Vol. 24, 1972, pp. 495-648.

[60] D. Lazarus, P. R. Scherer and D. R. Prothero, "Evolution of the Radiolarian Species-Complex Pterocanium: A Preliminary Survey," Journal of Paleontology, Vol. 59, No. 1, 1985, pp. 183-220, 29 Figs.

[61] J. P. Caulet, "Radiolaria from the Kerguelen Plateau, Leg119," In: J. Barron, B. Larsen, et al., Eds., Proceedings of the Ocean Drilling Program Scientific Results, Vol. 119, 1991, pp. 513-542. 